\title{
BILATERAL DYSGERMINOMA WITH PREGNANCY AND VIABLE BABY: A CASE REPORT.
}

Jyotsna Harlalka Kamra, Mandeep Kaur

1. Assistant Professor. Department of Obstetrics \& Gynaecology, Maharaja Agrasen Medical College, Agroha, Hisar.

2. Medical Officer. Gynaecologist, PCMS-1.

\section{CORRESPONDING AUTHOR}

Dr. Jyotsna Harlalka Kamra,

House no. 2420, Sec-14,

Hisar, Haryana,

E-mail: jyotsnakamra@gmail.com

Ph: 00919991981464

ABSTRACT: Dysgerminomas are the most common of primitive germ cell tumors of the ovary, accounting for $1-5 \%$ of all ovarian malignancies. The reproductive age group females are most commonly affected, thereby causing problems in conception and if pregnancy occurs, it leads to feto-maternal compromise. It is extremely rare to have a successful natural pregnancy, with viable child birth with a coexisting bilateral dysgerminoma, without any assisted reproductive interventions. We hereby report a case of successful spontaneous natural pregnancy, associated with bilateral dysgerminoma, with no feto-maternal compromise.

KEYWORDS: Dysgerminoma, Natural pregnancy, Viable birth

INTRODUCTION: Dysgerminomas are tumors originating within the primordial ovarian germ cells. They are very rare, frequently malignant, and are responsible for around $1 \%$ of all germ cell tumors. ${ }^{1,2}$ Around 20 to $25 \%$ of ovarian tumors originate in the germ cells and just $3 \%$ are malignant. ${ }^{3}$ Germ cell tumors account for around $70 \%$ of ovarian neoplasm cases, during the first decades of life, manifest malignant characteristics in $1 / 3$ of cases and are rarely found after this period. 3,4 Dysgerminoma has a classic correlation with seminoma of the testis, having an identical histological structure. 5 They are generally considered to have low malignant potential, but may spread if there is invasion through metastasis or capsule, or lymph node or blood cell involvement.

CASE REPORT: A 25-year female G4P2L1A1, who was referred from a peripheral civil hospital, came to casualty on 28/8/12 with term pregnancy with labor pains and ultrasonographically diagnosed B/L ovarian mass (? Malignant). According to USG report left sided ovarian mass was around 19x10 cm encroaching the pouch of Douglas and right-sided mass was around $6 \times 6 \mathrm{~cm}$ along with single intrauterine live fetus of 35 weeks gestation. She had previous two normal deliveries with one live issue and one spontaneous abortion. According to the patient she had no complaints previously viz, no menstrual abnormality, no constitutional symptoms like loss of appetite/fever, no bowel/bladder complaints, no abdominal swelling, and no family history of malignancy.

She conceived spontaneously. No ANC was done in this pregnancy. The only record available was the USG report, which had been done 1-week prior, showing 35-week POG live pregnancy with B/L? Malignant ovarian mass. 
On examination, pt. was poorly built and poorly nourished, pallor present. P/A: 34 wk, longitudinal lie, cephalic, FHR present and regular in rhythm, uterine contractions present.

$\mathrm{P} / \mathrm{V}$ : $\mathrm{Cx} 3 \mathrm{~cm}, \mathrm{PE}$, pushed anteriorly by mass (solid mass felt in POD) an immediate repeat USG was done to confirm the findings of the USG report which pt. was carrying with her. Our ultrasonographer reported it as the mass around $17 \mathrm{X} 19 \mathrm{~cm}$ lobulated solid appearance pushing cervix anteriorly with a provisional diagnosis of subserosal /cervical fibroid. Immediate routine investigations were done and pt. was prepared for LSCS with consent for hysterectomy with $\mathrm{B} / \mathrm{L}$ oophorectomy, in case the ovarian masses turned out to be malignant on gross appearance.

A midline laparotomy incision was given. LSCS was done and a live male baby of $2.2 \mathrm{~kg}$ was delivered. Grossly there was a huge solid mass present on left side around 20x10 $\mathrm{cm}$ and on right side there was $6 \times 6 \mathrm{~cm}$ solid mass present with intact capsule, without adhesions or cystic component. There were no peritoneal free fluid, no obvious peritoneal deposits present. On gross examination omentum, liver, bowel and undersurface of diaphragm was normal. There was no ovarian tissue that could be preserved and since the masses were solid, the family was completed; a decision for hysterectomy with B/L oophorectomy was taken.

Grossly: Right ovary was 9x6 cm and wt. 580 gm, Left ovary was 19x18x10 cm wt 1120 gm.

Histopathology: Suggestive of bilateral dysgerminoma of ovary with microscopic capsular and vascular invasion. Sections from bilateral fallopian tube and uterus were free from tumor cells.

A Post op CAT scan was done to find out/ rule out lymph node involvement and/or residual tumour. CT scan revealed no gross retroperitoneal lymphadenopathy, no free ascitic fluid. Viscera and chest were reported normal.

Staging of the tumour was done for deciding on need for chemotherapy/ radiotherapy. Grossly tumor was limited to the pelvis involving only both ovaries with normal bilateral fallopian tubes and uterus. (T3a) Negative lymph nodes on palpation and on CT scan. (N0). No e/o distant metastasis, grossly or on CT scan. (M0). Microscopic capsular and vascular invasion present (possibility of microscopic seeding of abdominal peritoneal surfaces). Staging llla is done on this basis. There is no facility of chemo/radiotherapy in our institution, so the patient was referred to higher centre for the same after discharge.

DISCUSSION: Dysgerminomas account for $1-5 \%$ of all ovarian malignancies in the first two decades of life. Approximately $80 \%$ of cases are reported in less than 30 years of age (mean: 21 years, a finding consistent with our case.) ${ }^{6}$ Several cases of pregnancies after treatment of dysgerminomas with various modalities including surgery and chemotherapy have been reported previously. Gershenson has reported that natural conception is possible in case of germ cell tumors of the ovary, a finding similar to our case. ${ }^{7}$ Hirota et al. in their study reported the frequency of ovarian tumor associated with pregnancy ranges from 1:80 to 1:2200 deliveries. ${ }^{8}$ While Ueda and Veki reported a single case $(0.9 \%)$ of dysgerminoma in their study on 106 cases of ovarian tumor surgically resected during pregnancy. ${ }^{9}$ But natural course of pregnancy in cases of dysgerminoma is extremely difficult, due to large sizes of the tumors, irregular menstruation, and collection of fluid as well as tubal adhesions.

Ovarian tumors generally remain asymptomatic, until they are discovered due to their large size or related complications. The patient in this report deserves special attention as she 
conceived naturally, without any assisted reproductive technique and spontaneous viable pregnancy and delivery was possible with a coexisting dysgerminoma.

The best outcome for both mother and child depends on early diagnosis and excision of the ovarian lesion while it is still intact. The pathologic type and extent of ovarian carcinoma seem to be the most important determining factors in the maternal prognosis. Several authors have stated that once the existence of ovarian malignancy is suspected, immediate laparotomy is indicated regardless of the stage of gestation. But Jubb, supports a more conservative approach in younger pregnant patients, especially if the ovarian lesion is intact or is of the pseudomucinous type. ${ }^{10}$ There still remain unsolved problems concerning conservative management before and after termination for early-stage ovarian malignancy associated with pregnancy. When we encounter FIGO stage-Ib or higher stage ovarian malignancies in the second trimester and the patient strongly wishes to continue with the pregnancy, very serious problems arise as to whether conservative surgery and chemotherapy should be performed in the gravid woman or not. Antineoplastic agents can be mutagenic or teratogenic, or cause fetal growth retardation or fetal death when used in the first trimester. ${ }^{11}$ However, Kim and Park, have documented the use of chemotherapeutic agents during the second trimester and delivery of a normal infant. ${ }^{12}$

Patterson et al. in their review of the close surveillance policy for stage I female germ cell tumors of the ovary, stated that five-year survival rate for Stage Ia dysgerminomas is over 95\%. ${ }^{13}$ As in our case histopathological examination showed capsular and micro vascular invasion due to which possibility of microscopic peritoneal implants could not be ruled out. Moreover bilaterality and big size further puts the patient to higher risk. So staging of IIIa was made and patient referred to higher centre for chemo radiotherapy. Until now only few cases of ovarian dysgerminoma during pregnancy have been described in literature. Mostly confined to one ovary, bilateral being extremely rare making it a noteworthy case.

DISCUSSION: Dysgerminomas account for $1-5 \%$ of all ovarian malignancies in the first two decades of life. Approximately $80 \%$ of cases are reported in less than 30 years of age (mean: 21 years, a finding consistent with our case.) ${ }^{6}$ Several cases of pregnancies after treatment of dysgerminomas with various modalities including surgery and chemotherapy have been reported previously. Gershenson has reported that natural conception is possible in case of germ cell tumors of the ovary, a finding similar to our case. ${ }^{7}$ Hirota et al. in their study reported the frequency of ovarian tumor associated with pregnancy ranges from 1:80 to 1:2200 deliveries. ${ }^{8}$ While Ueda and Veki reported a single case $(0.9 \%)$ of dysgerminoma in their study on 106 cases of ovarian tumor surgically resected during pregnancy. ${ }^{9}$ But natural course of pregnancy in cases of dysgerminoma is extremely difficult, due to large sizes of the tumors, irregular menstruation, and collection of fluid as well as tubal adhesions.

Ovarian tumors generally remain asymptomatic, until they are discovered due to their large size or related complications. The patient in this report deserves special attention as she conceived naturally, without any assisted reproductive technique and spontaneous viable pregnancy and delivery was possible with a coexisting dysgerminoma.

The best outcome for both mother and child depends on early diagnosis and excision of the ovarian lesion while it is still intact. The pathologic type and extent of ovarian carcinoma seem to be the most important determining factors in the maternal prognosis. Several authors have stated that once the existence of ovarian malignancy is suspected, immediate laparotomy is indicated regardless of the stage of gestation. But Jubb, supports a more conservative 
approach in younger pregnant patients, especially if the ovarian lesion is intact or is of the pseudomucinous type. ${ }^{10}$ There still remain unsolved problems concerning conservative management before and after termination for early-stage ovarian malignancy associated with pregnancy. When we encounter FIGO stage-Ib or higher stage ovarian malignancies in the second trimester and the patient strongly wishes to continue with the pregnancy, very serious problems arise as to whether conservative surgery and chemotherapy should be performed in the gravid woman or not. Antineoplastic agents can be mutagenic or teratogenic, or cause fetal growth retardation or fetal death when used in the first trimester. ${ }^{11}$ However, Kim and Park, have documented the use of chemotherapeutic agents during the second trimester and delivery of a normal infant. 12

Patterson et al. in their review of the close surveillance policy for stage I female germ cell tumors of the ovary, stated that five-year survival rate for Stage Ia dysgerminomas is over 95\%.13 As in our case histopathological examination showed capsular and micro vascular invasion due to which possibility of microscopic peritoneal implants could not be ruled out. Moreover bilaterality and big size further puts the patient to higher risk. So staging of IIIa was made and patient referred to higher centre for chemo radiotherapy. Until now only few cases of ovarian dysgerminoma during pregnancy have been described in literature. Mostly confined to one ovary, bilateral being extremely rare making it a noteworthy case.

CONCLUSION: The long term outcome patients with pure ovarian dysgerminoma is excellent Patients can be treated with fertility-sparing surgery and can expect good reproductive outcomes. A dysgerminoma confined to a both ovary, without ascites, although large may not metastasize or seed the peritoneal cavity/fluid or other pelvic/abdominal organs and a natural course of pregnancy with viable child birth may still be possible.

\section{REFERENCE:}

1. Ladis SH, Murray T, Bolden S, Wingo PA. Cancer statistics. CA Cancer J Clin. 1998;48:625.

2. Duska L, Bicher A. Câncer de ovário. In: Lambrou NC, Morse AN, Wallach E. Manual de ginecologia e obstetrícia do Johns Hopkins. Porto Alegre: Artmed; 1999. p.431-50.

3. Thimble EL. The NIH Consenus Conference on ovarian cancer: screening, treatment and follow-up. Gynecol Oncol. 1994;55:51-3.

4. Palenzuela G, Martin E, Meunier A, Beuzeboc P, Laurence V, Orbach D, et al. Comprehensive staging allows for excellent outcome in patients with localized malignant germ cell tumor of the ovary. Ann.Surg. 2008; 248:836-41.

5. Nguyen HN, Averette HE, Janicek M. Ovarian carcinoma: a review of the significance of familial risk factors and the role of prophylactic oophorectocmy in cancer prevention. Cancer. 1994; 74:545-55.

6. Bekaii-Saab T, Einhorn LH, Williams SD. Late relapse of ovarian dysgerminoma: case report and literature review. Gynecol Oncol 1999. Jan; 72(1):111-112. doi: 10.1006/gyno.1998.5207.

7. Gershenson DM. Update on malignant ovarian germ cell tumors. Cancer 1993. Feb;71(4)(Suppl):1581-1590. doi: 10.1002/cncr.2820710425.

8. Hirota Y, Sawada T, Kumayama M. Clinical study on ovarian malignant tumors associated with pregnancy. Rinsho Fujinka Sanka 1991;45:1331-1335. 
9. Ueda M, Ueki M. Ovarian tumors associated with pregnancy. Int J Gynaecol Obstet 1996. Oct;55(1):59-65. doi: 10.1016/0020-7292(96)02718-X.

10. Jubb ED. Primary ovarian carcinoma in pregnancy. Am J Obstet Gynecol 1963;85:345354.

11. Barber HR. Fetal and neonatal effects of cytotoxic agents. Obstet Gynecol 1981. Nov;58(5)(Suppl):41S-47S.

12. Kim DS, Park MI. Maternal and fetal survival following surgery and chemotherapy of endodermal sinus tumor of the ovary during pregnancy: a case report. Obstet Gynecol 1989. Mar;73(3 Pt 2):503-507.

13. Vicus D, Beiner ME, Klachook S, Le LW, Laframboise S, Mackay H. Pure dysgerminoma of the ovary 35 years on: a single institutional experience. Gynecol Oncol 2010. Apr;117(1):23-26. doi: 10.1016/j.ygyno.2009.12.024.

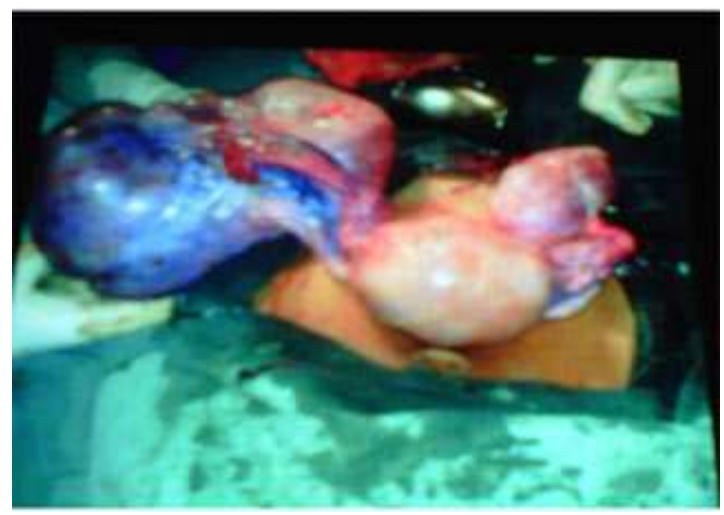

Fig. 1 A huge bilateral tumor with uterus delivery of baby by Lscs.

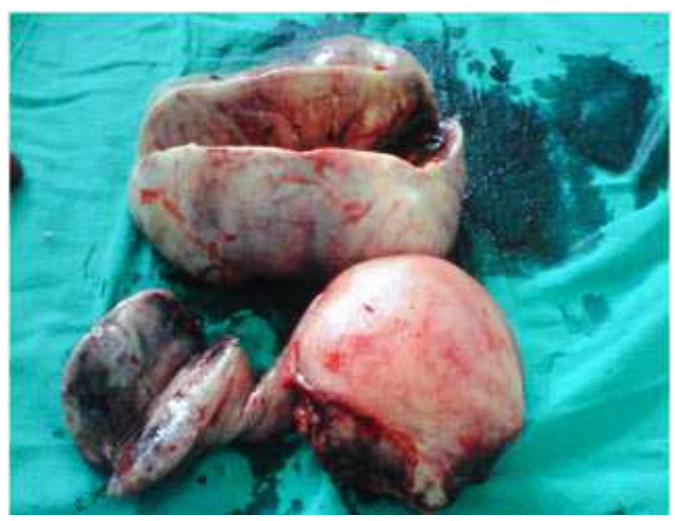

Fig. 2 Bilateral dysgerminoma tumor after and uterus after removal.

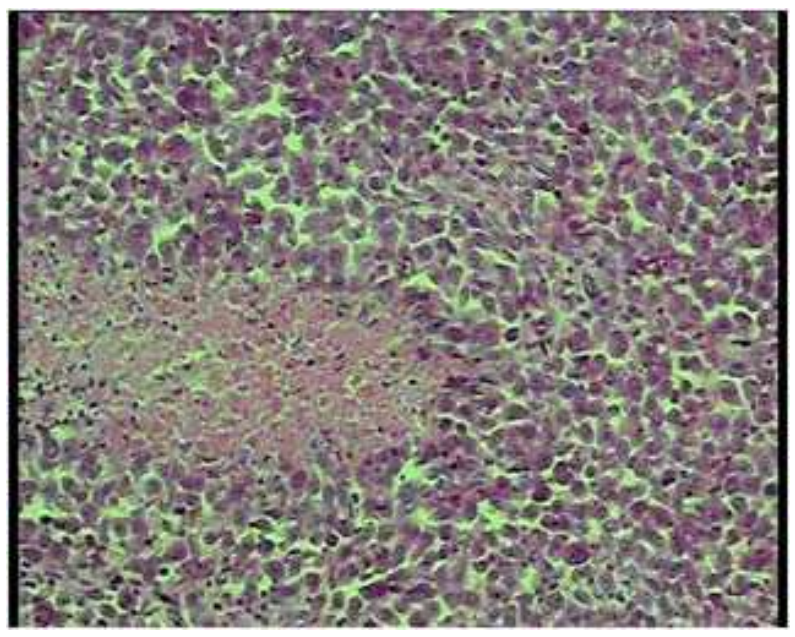

Fig. 3 Tissue section shows well defined nests of large uniform tumor cells with granular nuclear chromatin, prominent nucleoli and finely granular cytoplasm separated by fibrous strands containing lymphocytes. 